This item was submitted to Loughborough's Research Repository by the author.

Items in Figshare are protected by copyright, with all rights reserved, unless otherwise indicated.

\title{
Output-based disturbance rejection control for non-linear uncertain systems with unknown frequency disturbances using an observer backstepping approach
}

\section{PLEASE CITE THE PUBLISHED VERSION}

http://dx.doi.org/10.1049/iet-cta.2015.1160

\section{PUBLISHER}

(c) The Institution of Engineering and Technology

\section{VERSION}

AM (Accepted Manuscript)

\section{PUBLISHER STATEMENT}

This work is made available according to the conditions of the Creative Commons Attribution-NonCommercialNoDerivatives 4.0 International (CC BY-NC-ND 4.0) licence. Full details of this licence are available at: https://creativecommons.org/licenses/by-nc-nd/4.0/

\section{LICENCE}

CC BY-NC-ND 4.0

\section{REPOSITORY RECORD}

Yang, Jun, Zhengtao Ding, Wen-Hua Chen, and Shihua Li. 2016. "Output-based Disturbance Rejection Control for Non-linear Uncertain Systems with Unknown Frequency Disturbances Using an Observer Backstepping Approach". figshare. https://hdl.handle.net/2134/21958. 


\title{
Output-based disturbance rejection control for nonlinear uncertain systems with unknown frequency disturbances using an observer backstepping approach
}

\author{
Jun Yang a,*, Zhengtao Ding ${ }^{\mathrm{b}}$, Wen-Hua Chen ${ }^{\mathrm{c}}$, Shihua $\mathrm{Li}^{\mathrm{a}}$ \\ ${ }^{\text {a }}$ School of Automation, Southeast University, Key Laboratory of Measurement and Control of Complex Systems of \\ Engineering, Ministry of Education, Nanjing 210096, Jiangsu, China \\ ${ }^{\mathrm{b}}$ Control Systems Centre, School of Electrical and Electronic Engineering, University of Manchester, Sackville Street \\ Building, Manchester M13 9PL, UK
}

${ }^{\mathrm{c}}$ Department of Aeronautical and Automotive Engineering, Loughborough University, Leicestershire, LE11 3TU, UK

\begin{abstract}
This paper is concerned with the output feedback control design for a class of nonlinear uncertain systems subject to multiple sources of disturbances including model uncertainties, unknown constant disturbances, harmonic disturbances with unknown frequency and amplitude. The total disturbances and uncertainties are delicately represented by a compact exogenous model first. By incorporating the adaptive internal model principle, a set of dynamic estimators are developed for both state and disturbance observations. By means of observer backstepping technique, a composite output feedback controller is constructed based on the disturbance and state estimations. The stability of the closed-loop system is rigorously established based on Lyapunov stability criterion. A missile roll stabilization example is finally investigated to validate the effectiveness of the proposed control approach.
\end{abstract}

Key words: Output feedback control; Nonlinear uncertain system; Multiple disturbances and uncertainties; Internal model principle; Missile roll stabilization

\section{Introduction}

In general, the control performances and even stability of all industrial systems are severely jeopardized by various sources of disturbances and uncertainties [1-9]. It is not surprised that disturbance rejection and uncertainty attenuation is a crucial task in controller design. Due to such an urgent requirement, many advanced control approaches have been investigated to handle disturbances and uncertainties for nonlinear systems, such as stochastic control [10], nonlinear $H_{\infty}$ control [11], output regulation control [4,12], and sliding mode control [13], etc. Those control approaches improve robustness performance or disturbance rejection from different aspects. Among them, robust

\footnotetext{
* Corresponding author.

Email address: j.yang84@seu.edu.cn (Jun Yang).
} 
and adaptive control approaches have received a great deal of attentions in both theoretic society and industrial application sectors [4]. However, the way of robust control handling disturbances/uncertainties is generally recognized as a crude manner since it is a worst-case based design approach, while the adaptive control dealing with the uncertainties in a refined manner since the structured uncertainties are generally required $[1,14]$. Sliding mode control provides an active way to reject disturbances and uncertainties, but the resultant control chattering in practice restrains its applications in many industrial systems [13].

Recently, disturbance/uncertaity estimation and attenuation (DUEA) methods (including disturbance observerbased control (DOBC) [1], active disturbance rejection control (ADRC) [15], etc.) have been widely investigated as an alternative choice to achieve robustness against uncertainties as well as disturbance rejection performance (see $[1,2,5-7,15-23]$ and the references therein). Due to the two-degree-of-freedom design mechanism, DUEA performs well in striking a balance between the nominal performance and robustness $[1,18]$. Consequently, DUEA is generally regarded as a kind of refined robust control but crude adaptive control method [5]. Furthermore, DUEA method enable us to design a feedback controller and a disturbance estimator/compensator independently and then integrate them together. This property provides the flexibility of structure for DUEA as compared with output regulation control. In view of the promising properties, DUEA methods have considerable applications in various industrial sectors, such as mechatronic systems [16,22,24,25], aerospace systems [26-28], chemical and process systems [21].

In spite of the above significant advances in DUEA, most of above results aim at dealing with single disturbance or uncertainty $[2,29]$. However, in most practical engineering problems, the sources of disturbances and uncertainties are naturally multiple, and those multiple disturbances may exhibit distinct features and should be represented by different models and signals [2]. Taking the speed regulation problem of a permanent magnetic synchronous motor as an example, the unknown load torque would be a constant or bounded time-varying variable, the dead zone of inverter may cause harmonic speed ripple with unknown amplitude and frequency, and the inertial of moment could cause nonlinear uncertainties of the dynamics [24,25]. Clearly, those unknown uncertain factors have different features, and the existing DUEA approaches developed for a single disturbance is not capable of achieving higher control performances in the presence of multiple distinct disturbances and uncertainties [27].

Pioneered by Guo and his colleagues [2,29-31], a composite hierarchial anti-disturbance control (CHADC) approach has been proposed to handle multiple sources of disturbances and uncertainties on the basis of DUEA. For instance, in $[30,31]$, the disturbances and uncertainties are separated into two parts: one part is harmonic ones generated by an exogenous system while the other part is uncertainties supposed to satisfy the condition of $H_{2}$ norm-bounded. The idea behind CHADC approaches is to combine DOBC with $H_{\infty}$ or sliding mode control techniques for disturbance rejection and uncertainty attenuation. Further results have been proposed to handle the adaptive control problem for system under mismatched disturbances and uncertainties in [29]. However, most of existing CHADC approaches focus on state feedback control problem and few provide output feedback control results. In addition, most of existing CHADC approaches could only handle harmonic disturbances with unknown amplitude but with known frequency. The harmonic disturbances with unknown frequencies is still a design barrier for CHADC approaches. On the other hand, the internal model principle has been adopted for output regulation approach to compensate harmonic disturbances with unknown frequency [32,33]. Robust output regulation is further developed to suppress model uncertainties using robust control principle [4]. Due to the feature of worst-case-based design of robust control, the nominal control performance shall be sacrificed for robust output regulation of systems in the absence of model uncertainties.

In this paper, an output-based disturbance rejection control approach is proposed for nonlinear uncertain system subject to multiple sources of disturbances and uncertainties, particularly including model uncertainties and harmonic 
disturbances with unknown amplitude and frequency. Firstly, the multiple sources of disturbances are separated and represented by different models according to their distinct features. By means of adaptive internal model principle [34,32], adaptive state and disturbance observers are developed for both state and disturbance estimation design. A new output feedback disturbance rejection control law is then constructed for the nonlinear uncertain system under consideration by utilizing the observer backstepping design. A delicate stability result is established to show that the proposed composite control method could effectively attenuate the multiple disturbances including model uncertainties and external harmonic disturbance with unknown frequencies. An application to a missile roll stabilization problem is finally considered to demonstrate the effectiveness of the proposed method. The results validate the elegant robustness and disturbance rejection performance as claimed in the paper. The major properties of the proposed control approach are summarized as follows:

- The proposed control approach has made great progresses for DUEA to handle multiple disturbances/uncertainties, in particular, it provides an adequate way to handle both harmonic disturbances with unknown frequency and model uncertainties via an output feedback manner;

- Similar with ADRC, the proposed control approach does not require extensive model information of the plant; actually, only the order of the plant is demanded. Consequently, it can be deemed as an almost model free approach and an enhanced version of $A D R C$;

- As compared with output regulation approach, the proposed control approach deal with uncertainties via an estimation and compensation way, rather than a robust suppression manner.

The rest of the paper is organized as follows. In Section 2, the problem formulation of the paper is given. The detailed controller development of this paper is presented in Section 3, followed by the rigorous stability analysis of the closed-loop system in Section 4. A missile roll stabilization example is provided in Section 5 to validate the efficiency of the paper. Section 6 concludes the paper.

\section{Problem Formulation}

In this paper, we consider a class of single-input single-output nonlinear dynamic systems subject to multiple sources of disturbances and uncertainties, which is depicted as

$$
y^{(n)}(t)=f\left(y(t), \ldots, y^{(n-1)}(t), t\right)+b u(t)+d_{1}(t)
$$

where $y(t) \in \mathbb{R}$ is the controlled output, $u(t) \in \mathbb{R}$ is the control input, $d_{1}(t) \in \mathbb{R}$ is the external disturbance, $f\left(y(t), \ldots, y^{(n-1)}(t), t\right)$ denotes the unknown uncertain dynamics of the system, and $b$ is an unknown constant but with a known sign. The disturbances and uncertainties concerned here are from multiple sources:

- The external disturbance $d_{1}(t) \in \mathbb{R}$ is generated by the following exogenous system

$$
\dot{\sigma}(t)=S \sigma(t), d_{1}(t)=V \sigma(t)
$$

where $\sigma(t) \in \mathbb{R}^{s}$ denotes the internal state of the exogenous system. The external disturbance generated by exogenous system (2) represents a wide variety of disturbances in practical engineering systems. For instance, the unknown constant, ramp, harmonic disturbances have different characteristics. However, those signals and also the combination of them can be represented by the above exogenous system (2).

- The terms $f\left(y(t), \ldots, y^{(n-1)}(t), t\right)$ and bu(t) in (1) contain uncertain dynamics, which would cause adverse effects on the control performance and shall be considered in the control design. 
Letting $x_{i}(t)=y^{(i-1)}(t)$ for $i=1, \ldots, n$, the plant $(1)$ is represented as

$$
\dot{x}_{i}(t)=x_{i+1}(t), \dot{x}_{n}(t)=b_{0} u(t)+d_{1}(t)+d_{2}(t),
$$

with

$$
d_{2}(t)=f\left(x_{1}(t), \ldots, x_{n}(t), t\right)+\left(b-b_{0}\right) u(t),
$$

where $b_{0}$ is the nominal value of $b$, and $b_{0}$ and $b$ have the same sign. The following lemma is important and will be used in the later stability derivation.

Lemma 1 [35]: Consider a nonlinear system

$$
\dot{x}=f(x, u)
$$

with the state $x \in \mathbb{R}^{n}$ and the input $u \in \mathbb{R}$. If the following conditions are satisfied

- system $\dot{x}=f(x, u)$ is globally Input-to-State Stable (ISS),

- $u(t) \in \mathcal{L}_{\infty}$ and $\lim _{t \rightarrow \infty} u(t)=0$,

then the state $x$ of the system (4) tends to zero as $t$ goes to infinity, that is, $\lim _{t \rightarrow \infty} x(t)=0$.

\section{Design of Output-Based Disturbance Rejection Control}

\subsection{Design of State and Disturbance Observers}

Letting $x=\left[x_{1}, \ldots, x_{n}\right]^{T}$ and $d=d_{1}+d_{2}$, the nonlinear system (3) with additive disturbance governed by (2) is rewritten as

$$
\begin{aligned}
\dot{x}(t) & =A x(t)+B\left[b_{0} u(t)+d(t)\right], \\
\dot{w}(t) & =\bar{S} w(t)+\bar{W} \dot{d}_{2}(t), \\
d(t) & =\bar{V} w(t), y(t)=x_{1}(t)
\end{aligned}
$$

with

$$
\begin{gathered}
A=\left[\begin{array}{cccc}
0 & 1 & \cdots & 0 \\
\vdots & \vdots & \ddots & \vdots \\
0 & 0 & \cdots & 1 \\
0 & 0 & \cdots & 0
\end{array}\right], B=\left[\begin{array}{c}
0 \\
\vdots \\
0 \\
1
\end{array}\right], \bar{W}=\left[\begin{array}{c}
1 \\
0 \\
\vdots \\
0
\end{array}\right] \\
\bar{S}=\left[\begin{array}{ll}
0 & 0 \\
0 & S
\end{array}\right], \bar{V}=[1, V] .
\end{gathered}
$$

Consider the following state observers

$$
\begin{aligned}
& \dot{p}_{i}=p_{i+1}+\omega_{o}^{i} l_{i}\left(y-p_{1}\right), \quad i=1, \ldots, n-1, \\
& \dot{p}_{n}=b_{0} u+\omega_{o}^{n} l_{n}\left(y-p_{1}\right),
\end{aligned}
$$


and

$$
\begin{aligned}
& \dot{q}_{i}=q_{i+1}-\omega_{o}^{i} l_{i} q_{1}, \quad i=1, \ldots, n-1, \\
& \dot{q}_{n}=-\bar{V} w-\omega_{o}^{n} l_{n} q_{1},
\end{aligned}
$$

where $\omega_{o}>0$ is the observer bandwidth to be determined [36,37], and $l_{i}>0$ is the observer gain to be designed such that the polynomial $p_{0}(s)=s^{n}+l_{1} s^{n-1}+\cdots+l_{n-1} s+l_{n}$ is Hurwitz. The state $q_{i}$ is not implementable due to the unknown term of $\bar{V} w$.

Define the observer error as $\varepsilon_{i}=\left(x_{i}-p_{i}+q_{i}\right) / \omega_{o}^{i-1}$ for $i=1, \ldots, n$. A simple calculation gives

$$
\begin{aligned}
& \dot{\varepsilon}_{i}=\omega_{o}\left(\varepsilon_{i+1}-l_{i} \varepsilon_{1}\right), \quad i=1, \ldots, n-1, \\
& \dot{\varepsilon}_{n}=-\omega_{o} l_{n} \varepsilon_{1},
\end{aligned}
$$

or equivalently

$$
\dot{\varepsilon}=\omega_{o} A_{c} \varepsilon,
$$

with $\varepsilon=\left[\varepsilon_{1}, \ldots, \varepsilon_{n}\right]^{T}$ and

$$
A_{c}=\left[\begin{array}{cccc}
-l_{1} & 1 & \cdots & 0 \\
\vdots & \vdots & \ddots & \vdots \\
-l_{n-1} & 0 & \cdots & 1 \\
-l_{n} & 0 & \cdots & 0
\end{array}\right] .
$$

It is observed from (7) that the solution of state $q=\left[q_{1}, \ldots, q_{n}\right]^{\top}$ is in terms of disturbance $\bar{V} w$ through a stable filter. The lumped uncertainties contained in $d_{2}(t)$ are generally considered as bounded and even constant signals in most practical applications $[7,15,38]$. Consequently, there exists a $l \in R^{s+1}$ such that the second element of state $q$ can be represented as

$$
q_{2}=l^{\top} w .
$$

The variable in (10) is re-parameterized in a similar manner as [32]. For any known controllable pair $\{F, G\}$ with $F \in \mathbb{R}^{(s+1) \times(s+1)}$ being Hurwitz and $G \in \mathbb{R}^{s+1}$, there exists a $\psi \in \mathbb{R}^{s+1}$ and a $\phi \in \mathbb{R}^{s+1}$ such that

$$
\begin{aligned}
& \dot{\eta}=\left(F+G \psi^{\top}\right) \eta+\phi \dot{d}_{2}, \\
& q_{2}=\psi^{T} \eta .
\end{aligned}
$$

The benefit of the parameterization is illustrated as follows. Since the matrices $\bar{S}$ and $F$ have different eigenvalues, it can be shown that there exists a nonsingular matrix $M \in \mathbb{R}^{(s+1) \times(s+1)}$ which is the unique solution of the following Sylvester matrix [34]

$$
M \bar{S}-F M=G l^{\top} .
$$

Combining (11) and (12) we have $\eta=M w, \psi^{\top}=l^{\top} M^{-1}, \phi=M \bar{W}$. 
The observer for system (11) is designed as

$$
\dot{\xi}=F \xi+G p_{2}-F G y
$$

The estimation error is defined as $e=\xi-\eta-G y$. With $x_{2}=p_{2}-\psi^{\top} \eta+\omega_{o} \varepsilon_{2}$ in mind, it is obtained from (11) and (13) that

$$
\dot{e}=F e-\omega_{o} G \varepsilon_{2}-\phi \dot{d}_{2}
$$

Since $A_{c}$ and $F$ are Hurwitz matrices, there exist two positive definite matrices $P_{\varepsilon}=P_{\varepsilon}^{\top}$ and $P=P^{\top}$ satisfying

$$
A_{c}^{\top} P_{\varepsilon}+P_{\varepsilon} A_{c}=-2 I, F^{\top} P+P F=-2 I
$$

Consider the following Lyapunov function for the observation error dynamics (9) and (14)

$$
V_{0}(\varepsilon, e)=\frac{1}{2}\left[\beta \omega_{o} \varepsilon^{\top} P_{\varepsilon} \varepsilon+e^{\top} P e+\tilde{\psi}^{\top} \Gamma^{-1} \tilde{\psi}\right]
$$

where $\Gamma$ is a positive definite matrix, $\tilde{\psi}=\psi-\hat{\psi}$ and $\hat{\psi}$ is the estimate of $\psi$ to be designed later. Taking derivative of (16) along the observer error dynamics (9) and (14) gives

$$
\begin{aligned}
\dot{V}_{0}(\varepsilon, e)= & -\beta \omega_{o}^{2} \varepsilon^{\top} \varepsilon-e^{\top} e-\omega_{o} e^{\top} P G \varepsilon_{2} \\
& -e^{\top} P \phi \dot{d}_{2}-\tilde{\psi}^{\top} \Gamma^{-1} \dot{\hat{\psi}} \\
\leq & -\omega_{o}^{2}\left(\beta-\frac{1}{4 \kappa_{0}}\|P G\|^{2}\right) \varepsilon^{\top} \varepsilon \\
& -\left(1-\kappa_{0}\right) e^{\top} e-e^{\top} P \phi \dot{d}_{2}-\tilde{\psi}^{\top} \Gamma^{-1} \dot{\hat{\psi}}
\end{aligned}
$$

where $\kappa_{0}$ is a real positive constant.

\subsection{Construction of Output Feedback Composite Controller}

For brief expressions, the following variables are defined

$$
\begin{aligned}
& \delta_{\varepsilon, k}=\beta-\frac{1}{4 \kappa_{0}}\|P G\|^{2}-\sum_{j=1}^{k} \frac{1}{4 \kappa_{1, j}}, \\
& \delta_{e, k}=1-\kappa_{0}-\sum_{j=1}^{k} \kappa_{2, j}, \\
& \delta_{1, k}=d_{1}-\left\|\psi^{\top} G\right\|-\kappa_{1,1}-\frac{\|\psi\|^{2}}{4 \kappa_{2,1}}-\sum_{j=1}^{k} \kappa_{3, j}\left\|\psi^{\top} G\right\|^{2}, \\
& \delta_{j, k}=d_{j}-\kappa_{1, j}-\frac{\|\psi\|^{2}}{4 \kappa_{2, j}}-\frac{1}{4 \kappa_{3, j}},
\end{aligned}
$$

for $k=1, \ldots, n$ and $j=2, \ldots, k$ where we set $\kappa_{3,1}=0$ and all other parameters including $\kappa_{0}$ and $\kappa_{i, j}$ are positive reals to be determined latter. 
Initial Step: Define $\zeta_{1}=p_{1}-\alpha_{0}, \alpha_{0}=p_{1}-y$ and construct the Lyapunov function

$$
V_{1}\left(\varepsilon, e, \zeta_{1}\right)=V_{0}(\varepsilon, e)+\zeta_{1}^{2} / 2+\gamma_{1}^{-1} \tilde{d}_{1}^{2} / 2
$$

where $\tilde{d}_{1}=d_{1}-\hat{d}_{1}$ and $\gamma_{1}$ is a real positive design parameter. Define $\zeta_{2}=p_{2}-\alpha_{1}$ with $\alpha_{1}$ being a virtual control. A direct calculation gives

$$
\begin{aligned}
\dot{V}_{1} \leq & -\omega_{o}^{2}\left(\beta-\frac{1}{4 \kappa_{0}}\|P G\|^{2}\right) \varepsilon^{\top} \varepsilon \\
& -\left(1-\kappa_{0}\right) e^{\top} e-e^{\top} P \phi \dot{d}_{2}-\tilde{\psi}^{\top} \Gamma^{-1} \dot{\hat{\psi}} \\
& +\zeta_{1}\left(\zeta_{2}+\alpha_{1}-\psi^{\top} \eta+\omega_{o} \varepsilon_{2}\right)-\gamma_{1}^{-1} \tilde{d}_{1} \dot{\hat{d}}_{1} .
\end{aligned}
$$

Designing the virtual control

$$
\begin{aligned}
& \alpha_{1}=-c_{1} \zeta_{1}-\hat{d}_{1} \zeta_{1}+\hat{\psi}^{\top} \xi \\
& \dot{\hat{d}}_{1}=\gamma_{1} \zeta_{1}^{2}, \tau_{1}=\Gamma \frac{\partial \alpha_{0}}{\partial y} \xi \zeta_{1}
\end{aligned}
$$

yields

$$
\begin{aligned}
\dot{V}_{1} \leq & -\omega_{o}^{2}\left(\beta-\frac{1}{4 \kappa_{0}}\|P G\|^{2}\right) \varepsilon^{\top} \varepsilon-\left(1-\kappa_{0}\right) e^{\top} e \\
& -e^{\top} P \phi \dot{d}_{2}-\tilde{\psi}^{\top} \Gamma^{-1}\left(\dot{\hat{\psi}}-\tau_{1}\right)+\zeta_{1} \zeta_{2} \\
& -c_{1} \zeta_{1}^{2}-\left(d_{1}-\psi^{\top} G\right) \zeta_{1}^{2}+\psi^{\top} e \zeta_{1}+\omega_{0} \zeta_{1} \varepsilon_{2} \\
\leq & -\omega_{o}^{2} \delta_{\varepsilon, 1} \varepsilon^{\top} \varepsilon-\delta_{e, 1} e^{\top} e-e^{\top} P \phi \dot{d}_{2} \\
& -\tilde{\psi}^{\top} \Gamma^{-1}\left(\dot{\hat{\psi}}-\tau_{1}\right)+\zeta_{1} \zeta_{2}-c_{1} \zeta_{1}^{2}-\delta_{1,1} \zeta_{1}^{2} .
\end{aligned}
$$

The $2^{\text {nd }}$ Step: Define $\zeta_{3}=p_{3}-\alpha_{2}$ with $\alpha_{2}$ being the virtual control and choose the Lyapunov function

$$
V_{2}\left(\varepsilon, e, \zeta_{1}, \zeta_{2}\right)=V_{1}\left(\varepsilon, e, \zeta_{1}\right)+\zeta_{2}^{2} / 2+\gamma_{2}^{-1} \tilde{d}_{2}^{2} / 2
$$

Continue to design the virtual control $\alpha_{2}$ as follows

$$
\begin{aligned}
\alpha_{2}= & -c_{2} \zeta_{2}-\zeta_{1}-\omega_{o}^{2} l_{2}\left(y-p_{1}\right)-\hat{d}_{2}\left(\frac{\partial \alpha_{1}}{\partial y}\right)^{2} \zeta_{2} \\
& +\frac{\partial \alpha_{1}}{\partial y}\left(p_{2}-\hat{\psi} \xi\right)+\frac{\partial \alpha_{1}}{\partial \hat{d}_{1}} \dot{\hat{d}}_{1}+\frac{\partial \alpha_{1}}{\partial \xi} \dot{\xi}+\frac{\partial \alpha_{1}}{\partial \hat{\psi}} \tau_{2}
\end{aligned}
$$

with

$$
\dot{\hat{d}}_{2}=\gamma_{2}\left(\frac{\partial \alpha_{1}}{\partial y}\right)^{2} \zeta_{2}^{2}, \tau_{2}=\sum_{j=1}^{2} \Gamma \frac{\partial \alpha_{j-1}}{\partial y} \xi \zeta_{j}
$$


yields

$$
\begin{aligned}
\dot{V}_{2} \leq & -\omega_{o} \delta_{\varepsilon, 2} \varepsilon^{\top} \varepsilon-\delta_{e, 2} e^{\top} e-e^{\top} P \phi \dot{d}_{2} \\
& -\tilde{\psi}^{\top} \Gamma^{-1}\left(\dot{\hat{\psi}}-\tau_{2}\right)+\zeta_{2} \zeta_{3}-c_{1} \zeta_{1}^{2}-c_{2} \zeta_{2}^{2} \\
& -\delta_{1,2} \zeta_{1}^{2}-\delta_{2,2}\left(\frac{\partial \alpha_{1}}{\partial y}\right)^{2} \zeta_{2}^{2}+\frac{\partial \alpha_{1}}{\partial \hat{\psi}}\left(\dot{\hat{\psi}}-\tau_{2}\right) \zeta_{2}
\end{aligned}
$$

Inductive Step: Suppose at step $k$, there exist a smooth Lyapunov function $V_{k}\left(\varepsilon, e, \zeta_{1}, \ldots, \zeta_{k}\right)$ which is positive definite and proper, and a group of virtual controls $\alpha_{2}, \ldots, \alpha_{k}$ defined by

$$
\begin{aligned}
\alpha_{i}= & -c_{i} \zeta_{i}-\zeta_{i-1}-\omega_{o}^{i} l_{i}\left(y-p_{1}\right)-\hat{d}_{i}\left(\frac{\partial \alpha_{i-1}}{\partial y}\right)^{2} \zeta_{i} \\
& +\frac{\partial \alpha_{i-1}}{\partial y}\left(p_{2}-\hat{\psi} \xi\right)+\sum_{j=1}^{i-1} \frac{\partial \alpha_{i-1}}{\partial p_{j}} \dot{p}_{j}+\sum_{j=1}^{i-1} \frac{\partial \alpha_{i-1}}{\partial \hat{d}_{j}} \dot{\hat{d}}_{j} \\
& +\frac{\partial \alpha_{i-1}}{\partial \xi} \dot{\xi}+\frac{\partial \alpha_{i-1}}{\partial \hat{\psi}} \tau_{i}+\sum_{j=1}^{i-1} \frac{\partial \alpha_{j-1}}{\partial \hat{\psi}} \Gamma \frac{\partial \alpha_{i-1}}{\partial y} \xi \zeta_{j},
\end{aligned}
$$

where $\zeta_{i}=p_{i}-\alpha_{i-1}, \tilde{d}_{i}=d_{i}-\hat{d}_{i}$ and

$$
\dot{\hat{d}}_{i}=\gamma_{i}\left(\frac{\partial \alpha_{i-1}}{\partial y}\right)^{2} \zeta_{i}^{2}, \tau_{i}=\sum_{j=1}^{i} \Gamma \frac{\partial \alpha_{j-1}}{\partial y} \xi \zeta_{j},
$$

for $i=2, \ldots, k+1$ with $c_{i}>0$ being independent of the observer bandwidth $\omega_{o}$, such that

$$
\begin{aligned}
\dot{V}_{k} \leq & -\omega_{o}^{2} \delta_{\varepsilon, k} \varepsilon^{\top} \varepsilon-\delta_{e, k} e^{\top} e-e^{\top} P \phi \dot{d}_{2} \\
& -\tilde{\psi}^{\top} \Gamma^{-1}\left(\dot{\hat{\psi}}-\tau_{k}\right)+\zeta_{k} \zeta_{k+1}-\sum_{j=1}^{k} c_{j} \zeta_{j}^{2} \\
& -\sum_{j=1}^{k} \delta_{j, k}\left(\frac{\partial \alpha_{j-1}}{\partial y}\right)^{2} \zeta_{j}^{2}+\sum_{j=1}^{k} \frac{\partial \alpha_{j-1}}{\partial \hat{\psi}}\left(\dot{\hat{\psi}}-\tau_{k}\right) \zeta_{j}
\end{aligned}
$$

We consider now the Lyapunov function candidate

$$
\begin{aligned}
& V_{k+1}\left(\varepsilon, e, \zeta_{1}, \ldots, \zeta_{k+1}\right) \\
& \quad=V_{k}\left(\varepsilon, e, \zeta_{1}, \ldots, \zeta_{k}\right)+\zeta_{k+1}^{2} / 2+\gamma_{k+1}^{-1} \tilde{d}_{k+1}^{2} / 2,
\end{aligned}
$$

where $\zeta_{k+1}=p_{k+1}-\alpha_{k}$. The following proposition is important for the following construction, and the detailed proof can be found from the appendix. 
Proposition 1: The following condition is satisfied

$$
\begin{aligned}
& \frac{d}{d t}\left(\frac{1}{2} \zeta_{k+1}^{2}+\frac{1}{2 \gamma_{k+1}} \tilde{d}_{k+1}^{2}\right) \\
& \leq \zeta_{k+1}\left[\zeta_{k+2}-c_{k+1} \zeta_{k+1}-\zeta_{k}-\frac{\partial \alpha_{k}}{\partial \hat{\psi}}\left(\dot{\hat{\psi}}-\tau_{k+1}\right)\right. \\
& \left.\quad+\sum_{j=1}^{k} \frac{\partial \alpha_{j-1}}{\partial \hat{\psi}} \Gamma \frac{\partial \alpha_{k}}{\partial y} \xi \zeta_{j}\right]+\kappa_{2, k+1} e^{\top} e \\
& \quad+\tilde{\psi}^{\top} \frac{\partial \alpha_{k}}{\partial y} \xi \zeta_{k+1}+\kappa_{3, k+1}\left\|\psi^{\top} G\right\|^{2} \zeta_{1}^{2} \\
& \quad+\frac{\omega_{o}^{2}}{4 \kappa_{1, k+1}} \varepsilon^{\top} \varepsilon-\delta_{1, k+1}\left(\frac{\partial \alpha_{k}}{\partial y}\right)^{2} \zeta_{k+1}^{2}
\end{aligned}
$$

for the dynamic systems under consideration.

Putting (25), (26) and (27) together we have

$$
\begin{aligned}
\dot{V}_{k+1} \leq & -\omega_{o}^{2} \delta_{\varepsilon, k+1} \varepsilon^{\top} \varepsilon-\delta_{e, k+1} e^{\top} e-e^{\top} P \phi \dot{d}_{2} \\
& -\tilde{\psi}^{\top} \Gamma^{-1}\left(\dot{\hat{\psi}}-\tau_{k+1}\right)+\zeta_{k+1} \zeta_{k+2} \\
& -\sum_{j=1}^{k+1} c_{j} \zeta_{j}^{2}-\sum_{j=1}^{k+1} \delta_{j, k+1}\left(\frac{\partial \alpha_{j-1}}{\partial y}\right)^{2} \zeta_{j}^{2} \\
& +\sum_{j=1}^{k+1} \frac{\partial \alpha_{j-1}}{\partial \hat{\psi}}\left(\dot{\hat{\psi}}-\tau_{k+1}\right) \zeta_{j}
\end{aligned}
$$

This completes the inductive argument. Continue to apply the inductive argument, and at the $n$th step the real controller is designed as

$$
u=\alpha_{n} / b_{0},
$$

with

$$
\dot{\hat{\psi}}=\tau_{n}
$$

resulting in

$$
\begin{aligned}
\dot{V}_{n} \leq & -\omega_{o}^{2} \delta_{\varepsilon, n} \varepsilon^{\top} \varepsilon-\delta_{e, n} e^{\top} e-\sum_{j=1}^{n} c_{j} \zeta_{j}^{2} \\
& -\sum_{j=1}^{n} \delta_{j, n}\left(\frac{\partial \alpha_{j-1}}{\partial y}\right)^{2} \zeta_{j}^{2}-e^{\top} P \phi \dot{d}_{2} .
\end{aligned}
$$




\section{Stability Analysis of the Closed-loop System}

Observe that it is possible to set the constant positive reals $\kappa_{0}, \kappa_{i, j}$, and $d_{j}, i=1,2,3$ and $j=1, \ldots, n$ such that $\delta_{\varepsilon, n}>0, \delta_{e, n}>0, \delta_{j, n}>0, j=1, \ldots, n$. With those conditions in mind, we have

$$
\dot{V}_{n} \leq-\omega_{o}^{2} \delta_{\varepsilon} \varepsilon^{\top} \varepsilon-\delta_{e} e^{\top} e-\sum_{j=1}^{n} c_{j} \zeta_{j}^{2}-e^{\top} P \phi \dot{d}_{2}
$$

Theorem 1: Suppose that the derivative of disturbance $d_{2}(t)$ is bounded, i.e., $\dot{d}_{2}(t) \in \mathcal{L}_{\infty}$. Taking $\dot{d}_{2}(t)$ as an input of the the plant (5), the proposed composite control law guarantees the ISS of the closed-loop systems consisting of the plant (5), the control law (29), and the filters (6) and (13), that is, $x_{i}(t) \in \mathcal{L}_{\infty}, i=1, \ldots, n$.

Proof: With the observation errors (9) and (14) in mind, calculating the dynamic behaviors of $\zeta_{i}$, the closed-loop system is governed by

$$
\begin{aligned}
\dot{\zeta}_{1}= & \zeta_{2}-c_{1} \zeta_{1}-\hat{d}_{1} \zeta_{1}+\left(\omega_{o} \varepsilon_{2}-\psi^{\top} \eta+\hat{\psi} \xi\right) \\
\dot{\zeta}_{i}= & \zeta_{i+1}-c_{i} \zeta_{i}-\zeta_{i-1}-\hat{d}_{i}\left(\frac{\partial \alpha_{i-1}}{\partial y}\right)^{2} \zeta_{i} \\
& +\frac{\partial \alpha_{i-1}}{\partial y}\left(\omega_{o} \varepsilon_{2}-\psi^{\top} \eta+\hat{\psi} \xi\right) \\
& -\sum_{j=i+1}^{n} \frac{\partial \alpha_{i-1}}{\partial \hat{\psi}} \Gamma \frac{\partial \alpha_{j-1}}{\partial y} \xi \zeta_{j} \\
& +\sum_{j=2}^{i-1} \frac{\partial \alpha_{j-1}}{\partial \hat{\psi}} \Gamma \frac{\partial \alpha_{i-1}}{\partial y} \xi \zeta_{j}, i=2, \ldots, n \\
\dot{\varepsilon}= & \omega_{o} A_{c} \varepsilon \\
\dot{e}= & F e-\omega_{o} G \varepsilon_{2}-\phi \dot{d}_{2}
\end{aligned}
$$

Based on (31), it is straightforward to show that the close-loop system (32) in the absence of perturbation $\dot{d}_{2}(t)$ is asymptotically stable. Consequently, the genuine closed-loop system (32) can actually be considered as an asymptotically stable system perturbed by a bounded input signal $\phi \dot{d}_{2}(t)$. The conclusion that $x_{i}(t) \in \mathcal{L}_{\infty}, i=1, \ldots, n$ directly follows from the ISS property.

Theorem 2: Suppose that the disturbance $\dot{d}_{2}(t)$ is bounded and tends to zero as t goes to infinity, i.e., $\dot{d}_{2}(t) \in \mathcal{L}_{\infty}$ and $\lim _{t \rightarrow \infty} \dot{d}_{2}(t)=0$. The proposed composite control law guarantees the asymptotical stability of the closed-loop systems consisting of the plant (5), the control law (29), and the filters (6) and (13), that is, $x_{i}(t) \in \mathcal{L}_{\infty}, i=1, \ldots, n$ and $\lim _{t \rightarrow \infty} x_{i}(t)=0$.

Proof: With the assumption on $\dot{d}_{2}(t)$ in mind, it follows from Lemma 1 and Theorem 1 that

$$
\lim _{t \rightarrow \infty} \zeta_{i}(t)=0, \lim _{t \rightarrow \infty} \varepsilon(t)=0, \quad \lim _{t \rightarrow \infty} e(t)=0
$$


In a similar manner with [32], we can derive the results that

$$
\lim _{t \rightarrow \infty} \hat{\psi}(t)=\psi
$$

if the external disturbance $\sigma(t)$ contains components at $s / 2$ distinct frequencies. Define $\hat{x}_{i}=\left(p_{i}-q_{i}\right) / \omega_{o}^{i-1}$ for $i=1, \ldots, n$. Combining (6) and (7), we have

$$
\dot{\hat{x}}_{i}(t)=\omega_{o}\left[\hat{x}_{i+1}+l_{i}\left(y-\hat{x}_{1}\right)\right]
$$

Note that the conditions $\lim _{t \rightarrow \infty} x_{1}(t)=0$ and $\lim _{t \rightarrow \infty} \varepsilon_{1}(t)=0$ imply that $\lim _{t \rightarrow \infty} \hat{x}_{1}(t)=0$. It follows from the Barbalat Lemma that $\lim _{t \rightarrow \infty} \hat{x}_{i}(t)=0$ for $i=2, \ldots, n$. This completes the proof.

Remark 1: The assumption that $\dot{d}_{2}$ is bounded is used for rigorous stability analysis. In general, the lumped disturbances may contain some state variables, and it is difficult to prove the stability for this general case of $d_{2}(t)=f(x, t)$ $[1,6,7,15]$. One may doubt the availability of the proposed method in this case. In many practical engineering systems, the dominated dynamics can be stabilized by the feedback control, and the state uncertainties contained in the lumped disturbance $d_{2}(t)$ are relatively weak, which will not affect the system stability. In this case, such uncertainties can be regarded as part of the lumped disturbance and can be handled by the proposed method. This is possibly the major reason for the prevalence of using the disturbance estimator-based control to compensate plant uncertainties in real engineering systems.

For the convenience of the readers, the parameter tuning guidelines of the proposed control approach are summarized as follows:

- Observer Parameters: 1) Choose a controllable pair $\{F, G\}$ with $F$ being Hurwitz; 2) Select observer gains $l_{i}>0$ such that the polynomial $p_{0}(s)=s^{n}+l_{1} s^{n-1}+\cdots+l_{n-1} s+l_{n}$ is Hurwitz; and 3) Design a positive definite matrix $\Gamma$ and positive parameters $\gamma_{i}>0$ for $i=1, \ldots, n$.

- Control Parameters: The feedback control parameters are designed such that $c_{i}>0$ for $i=1, \ldots, n$.

\section{Application to A Missile Roll Stabilization Example}

The roll stabilization for a generic PGM (precision guided munitions) missiles is investigated in this section to demonstrate the performance of the proposed method in this paper. The dynamics of the missile in roll plane, ignoring the flexible mode, is described as follows [39]

$$
\ddot{\phi}=C_{\alpha} \sin 4 \phi-w_{R R} \dot{\phi}+K_{\delta} \delta_{a}+d(t)
$$

where $\phi$ and $\dot{\phi}$ are roll angle and roll rate of PGM missiles, $\delta_{a}$ is fin deflection. $C_{\alpha}, w_{R R}$, and $K_{\delta}$ are the disturbance coefficient, roll rate bandwidth and fin effectiveness, respectively. $d(t)$ is external disturbance possibly caused by wind gust. The nominal parameters of $C_{\alpha}, w_{R R}$ and $K_{\delta}$ are taken as $C_{\alpha 0}=70, w_{R R 0}=5$ and $K_{\delta 0}=1450$, respectively.

Note that the roll dynamics would be interrupted by multiple sources of disturbances including external disturbances and internal model uncertainties. The external disturbance $d(t)$ may be represented by unknown constant, harmonic signal with unknown frequency and amplitude, and so on. The model uncertainties are largely induced by parameter 
perturbations of $C_{\alpha}$ and $K_{\delta}$. The objective of controller design is to achieve robust altitude stabilization of the missile under various disturbances and uncertainties.

\subsection{Controller Development}

Letting $y(t)=\phi(t), u(t)=\delta_{a}(t), b=K_{\delta}$, and $f(y(t), \dot{y}(t), t)=C_{\alpha} \sin 4 y(t)-w_{R R} \dot{y}(t)$ the roll dynamics can be formulated as (1). To facilitate the controller design, the following intermediate variables are defined

$$
z_{1}=y, \alpha_{1}=-\left(c_{1}+\hat{d}_{1}\right) z_{1}+\hat{\psi}^{\top} \xi, z_{2}=p_{2}-\alpha_{1},
$$

where $\hat{d}_{1}, \hat{d}_{2}$ and $\hat{\psi}$ are adaptive variables to be updated.

The observers and adaptive dynamics are designed as

$$
\begin{aligned}
\dot{p}_{1} & =p_{2}+k_{1}\left(y-p_{1}\right), \\
\dot{p}_{2} & =b_{0} u+k_{2}\left(y-p_{1}\right), \\
\dot{\xi} & =F \xi+G p_{2}-F G y, \\
\dot{\hat{d}}_{1} & =\gamma_{1} y^{2}, \dot{\hat{d}}_{2}=\gamma_{2}\left(c_{1}+\hat{d}_{1}\right)^{2} z_{2}^{2}, \\
\dot{\hat{\psi}} & =-\Gamma \xi z_{1}-\Gamma\left(c_{1}+\hat{d}_{1}\right) \xi z_{2},
\end{aligned}
$$

where $b_{0}=K_{\delta 0}$. The composite controller is finally designed as

$$
\begin{aligned}
u= & \left\{-z_{1}-\left[c_{2}+\hat{d}_{2}\left(c_{1}+\hat{d}_{1}\right)^{2}+\xi^{\top} \Gamma\left(c_{1}+\hat{d}_{1}\right) \xi\right] z_{2}\right. \\
& -k_{2}\left(y-p_{1}\right)-\left(c_{1}+\hat{d}_{1}\right)\left(p_{2}-\hat{\psi}^{\top} \xi\right)-\gamma_{1} z_{1}^{3} \\
& \left.+\hat{\psi}^{\top}\left(F \xi+G p_{2}-F G z_{1}\right)-\xi^{\top} \Gamma \xi z_{1}\right\} / b_{0} .
\end{aligned}
$$

The controller parameters of (34) and (35) for the roll stabilization problem of (33) are assigned as follows

$$
\begin{gathered}
k_{1}=2, k_{2}=1, \gamma_{1}=1, \gamma_{2}=1, c_{1}=1, c_{2}=1, \\
F=\left[\begin{array}{ccc}
0 & 1 & 0 \\
0 & 0 & 1 \\
-3.375 & -6.75 & -4.5
\end{array}\right], G=\left[\begin{array}{l}
0 \\
1 \\
1
\end{array}\right], \Gamma=\left[\begin{array}{ccc}
50 & 0 & 0 \\
0 & 50 & 0 \\
0 & 0 & 50
\end{array}\right] .
\end{gathered}
$$

\subsection{Simulation Results}

In this part, we will investigate the robustness performance of the proposed composite approach against multiple sources of disturbances and uncertainties. As shown in Table 5.2, three cases of perturbations on fin effectiveness $K_{\delta}$ and disturbance coefficient $C_{\alpha}$ as well as external disturbances $d_{1}$ are taken into account in this part.

To evaluate the performance of the proposed composite controller, a basic ADRC approach [15] is employed here as a benchmark disturbance rejection approach for comparison study. The extended state observer of the basic ADRC 
Table 1

Simulation Scenarios Setting for Performance Test

\begin{tabular}{|c|c|c|c|}
\hline Simulation Scenarios & $K_{\delta}$ & $C_{\alpha}$ & $d_{1}$ \\
\hline Case I & 1450 & 70 & 1000 \\
\hline Case II & 800 & 35 & $-400 \sin (4 \pi t)-800$ \\
\hline Case III & 2000 & 105 & $500 \sin (5 \pi t)+1000$ \\
\hline
\end{tabular}

is designed as

$$
\begin{aligned}
& \dot{\chi}_{1}=\chi_{2}-\beta_{1}\left(\chi_{1}-y\right), \\
& \dot{\chi}_{2}=b_{0} u+\chi_{3}-\beta_{2}\left(\chi_{1}-y\right), \\
& \dot{\chi}_{3}=-\beta_{3}\left(\chi_{1}-y\right)
\end{aligned}
$$

and the control law is designed as

$$
u=\frac{k_{1} \chi_{1}+k_{2} \chi_{2}-\chi_{3}}{b_{0}}
$$

where the parameters are chosen as $\beta_{1}=120, \beta_{2}=4800, \beta_{3}=64000, k_{1}=-400$ and $k_{2}=-40$, respectively.

The response curves of the roll angle, roll rate, and fin deflection under the proposed composite control law (35) and the basic ADRC (37) in the presence of three cases of multiple disturbances and uncertainties described in Table 5.2 are shown in Figs. 1-3, respectively.

As shown by Fig. 1, the basic ADRC approach could effectively compensate the lumped disturbance effects caused by model uncertainties and constant external disturbances. However, it is shown by Figs. 2 and 3 that the basic ADRC fails to precisely compensate the effects of harmonic disturbances. On the contrary, as clearly shown by Figs. 1-3, the proposed composite control law (35) can effectively attenuation the multiple disturbances including unknown constant disturbance, harmonic disturbance with unknown amplitude and frequency, model uncertainties caused by unknown parameters of $K_{\delta}$ and $C_{\alpha}$.

\section{Conclusions}

The output feedback control design problem of a class of nonlinear systems subject to multiple sources of disturbances/uncertainties including model uncertainties, constant and harmonic external disturbances has been investigated in the paper. By using adaptive internal model principle and observer backstepping technique, a new composite output feedback controller has been developed for multiple disturbance estimation and compensation. The proposed method has inherited many advantages of DUEA, CHADC and output regulation approaches. The effectiveness and feasibility of the proposed approach has been validated by application to a missile roll stabilization example. The future work will focus on quantitative robustness analysis and stochastic systems with the proposed control method.

\section{Acknowledgements}

This work was supported in part by National Natural Science Foundation of China under Grants 61203011, 61473080 and 61403227, PhD Program Foundation of Ministry of Education of China under Grant 20120092120031, and Natural Science Foundation of Jiangsu Province under Grant BK2012327. 

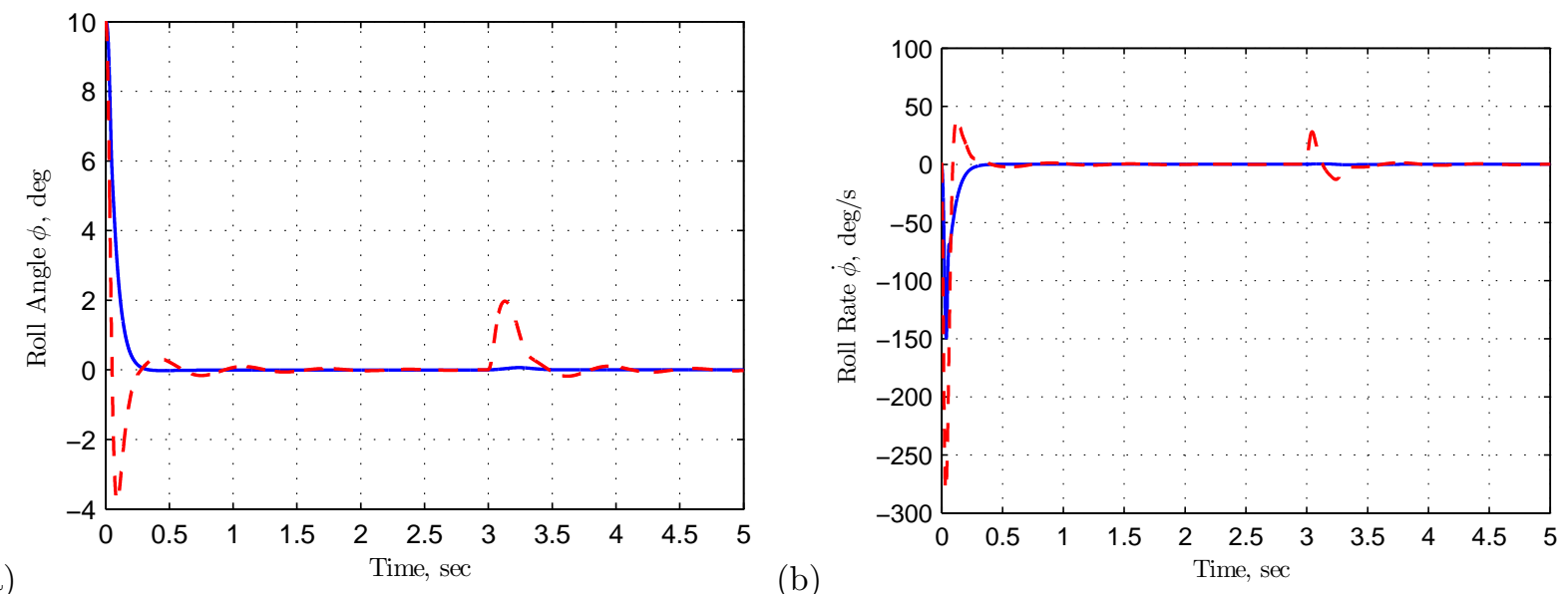

(a)

(b)

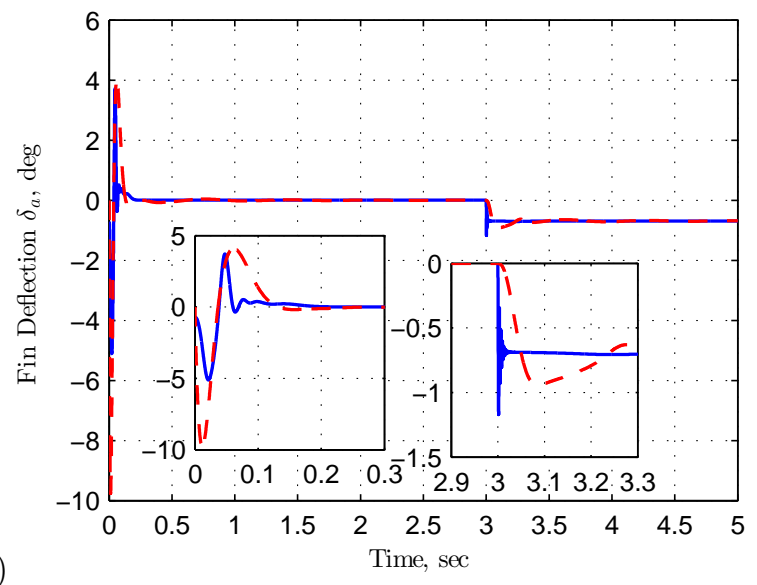

Fig. 1. Robustness performance test results of roll stabilization problem in the presence of disturbances and uncertainties in Case I under the proposed method (solid blue) and the basic ADRC method (dashed red): (a) roll angle; (b) roll rate; (c) fin deflection.

\section{References}

[1] S.H. Li, J. Yang, W.-H. Chen, and X.S. Chen. Disturbance Observer Based Control: Methods and Applications. CRC Press, Florida, USA, 2014.

[2] L. Guo and S. Cao. Anti-disturbance control theory for systems with multiple disturbances: A survey. ISA transactions, 53(4):846$849,2014$.

[3] Z.T. Ding. Nonlinear and Adaptive Control Systems. IET, London, 2013.

[4] Z. Chen and J. Huang. Stabilization and Regulation of Nonlinear Systems: A Robust and Adaptive Approach. Springer, London, 2015.

[5] W.-H. Chen, J. Yang, L. Guo, and S.H. Li. Disturbance observer-based control and related methods-an overview. IEEE Transactions on Industrial Electronics, DOI:10.1109/TIE.2015.2478397, 2015.

[6] Z. Gao. On the centrality of disturbance rejection in automatic control. ISA Transactions, 4(53):850-857, 2014.

[7] Y. Huang and W.C. Xue. Active disturbance rejection control: methodology and theoretical analysis. ISA transactions, 53(4):963976, 2014.

[8] Y. Li, S. Tong, and T. Li. Observer-based adaptive fuzzy tracking control of mimo stochastic nonlinear systems with unknown control direction and unknown dead-zones. IEEE Transactions on Fuzzy Systems, 4(23):1228-1241, 2015.

[9] S. Tong, S. Sui, and Y. Li. Fuzzy adaptive output feedback control of mimo nonlinear systems with partial tracking errors constrained. IEEE Transactions on Fuzzy Systems, 4(23):729-742, 2015.

[10] K.J. Astrom. Introduction to Stochastic Control Theory. Academic Press, NY, USA, 1970. 

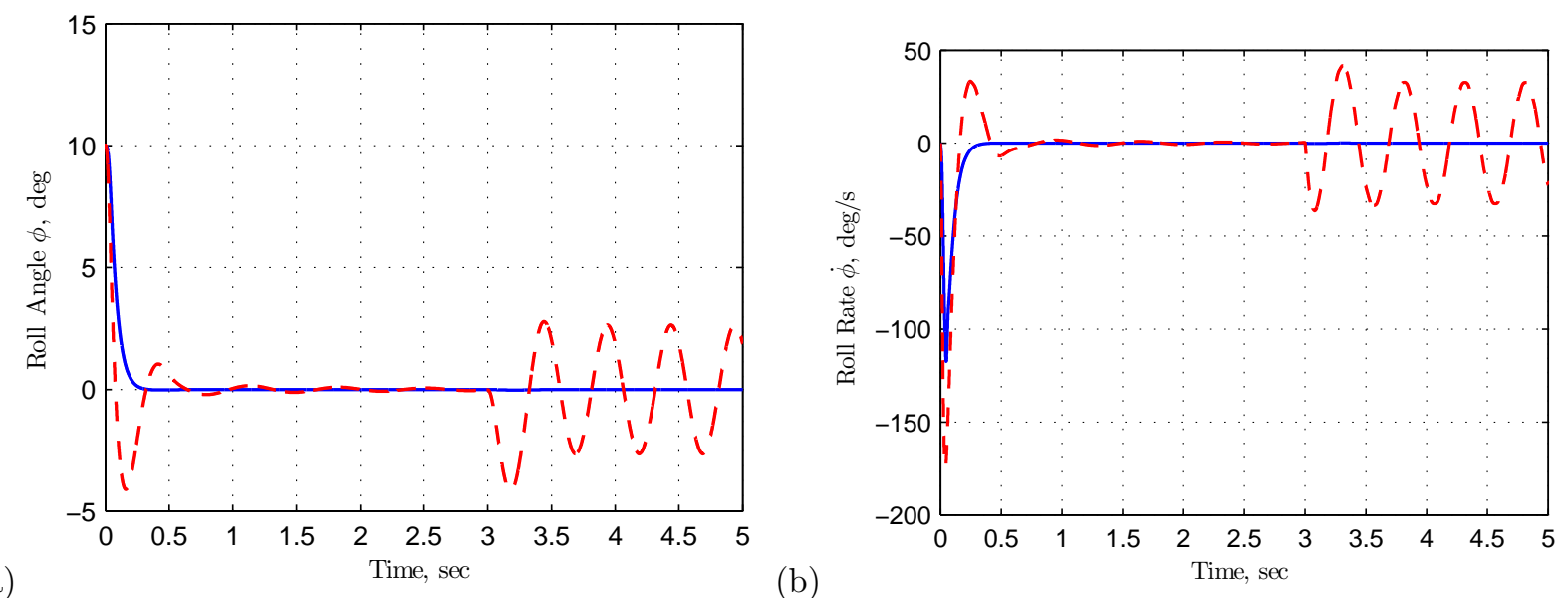

(a)

(b)

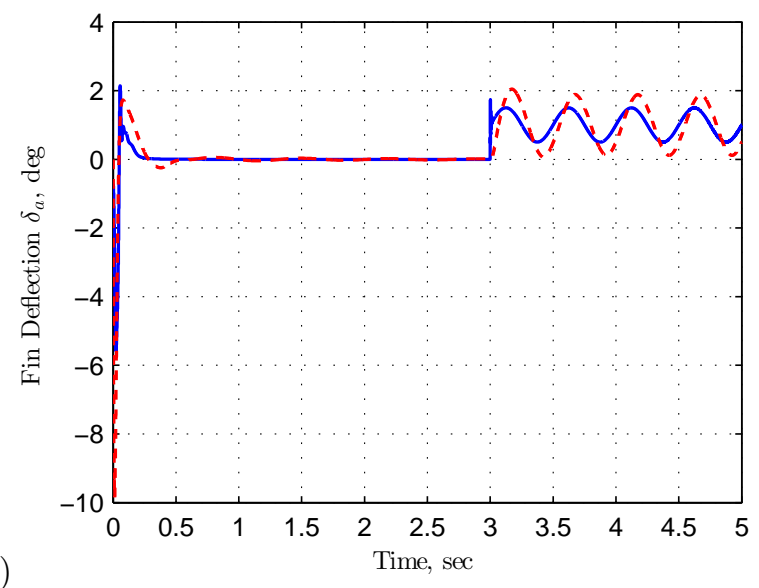

Fig. 2. Robustness performance test results of roll stabilization problem in the presence of disturbances and uncertainties in Case II under the proposed method (solid blue) and the basic ADRC method (dashed red): (a) roll angle; (b) roll rate; (c) fin deflection.

[11] K.M. Zhou and J.C. Doyle. Essentials of robust control. Prentice Hall, 1998.

[12] A. Isidori and C.I. Byrnes. Output regulation of nonlinear systems. IEEE Transactions on Automatic Control, 35(2):131-140, 1990.

[13] V.I. Utkin. Variable structure systems with sliding modes. IEEE Transactions on Automatic Control, AC-22(2):212-222, 1977.

[14] J. Yang, W.-H. Chen, and S.H. Li. Non-linear disturbance observer-based robust control for systems with mismatched disturbances/uncertainties. IET Control Theory and Application, 5(18):2053-2062, 2011.

[15] J. Han. From PID to active disturbance rejection control. IEEE Transactions on Industrial Electronics, 56(3):900-906, 2009.

[16] J.H. She, X. Xin, and Y.D. Pan. Equivalent-input-disturbance approach-Analysis and application to disturbance rejection in dualstage feed drive control system. IEEE Transactions on Mechatronics, 16(2):330-340, 2011.

[17] Q.-C. Zhong, A. Kuperman, and R.-K. Stobart. Design of UDE-based controllers from their two-degree-of-freedom nature. International Journal of Robust and Nonlinear Control, 17(21):1994-2008, 2011.

[18] H. Shim and N.H. Jo. An almost necessary and sufficient condition for robust stability of closed-loop systems with disturbance observer. Automatica, 45(1):296-299, 2009.

[19] X. Chen, S. Komada, and T. Fukuda. Design of a nonlinear disturbance observer. IEEE Transactions on Industrial Electronics, 37(2):429-436, 2000.

[20] E. Sariyildiz and K. Ohnishi. A guide to design disturbance observer. Journal of Dynamic Systems, Measurment, and Control, 2(136):021011, 2014.

[21] P. Zhou, W. Dai, and T.Y. Chai. Multivariable disturbance observer based advanced feedback control design and its application to a grinding circuit. IEEE Transactions on Control Systems Technology, 4(22):1471-1485, 2014. 

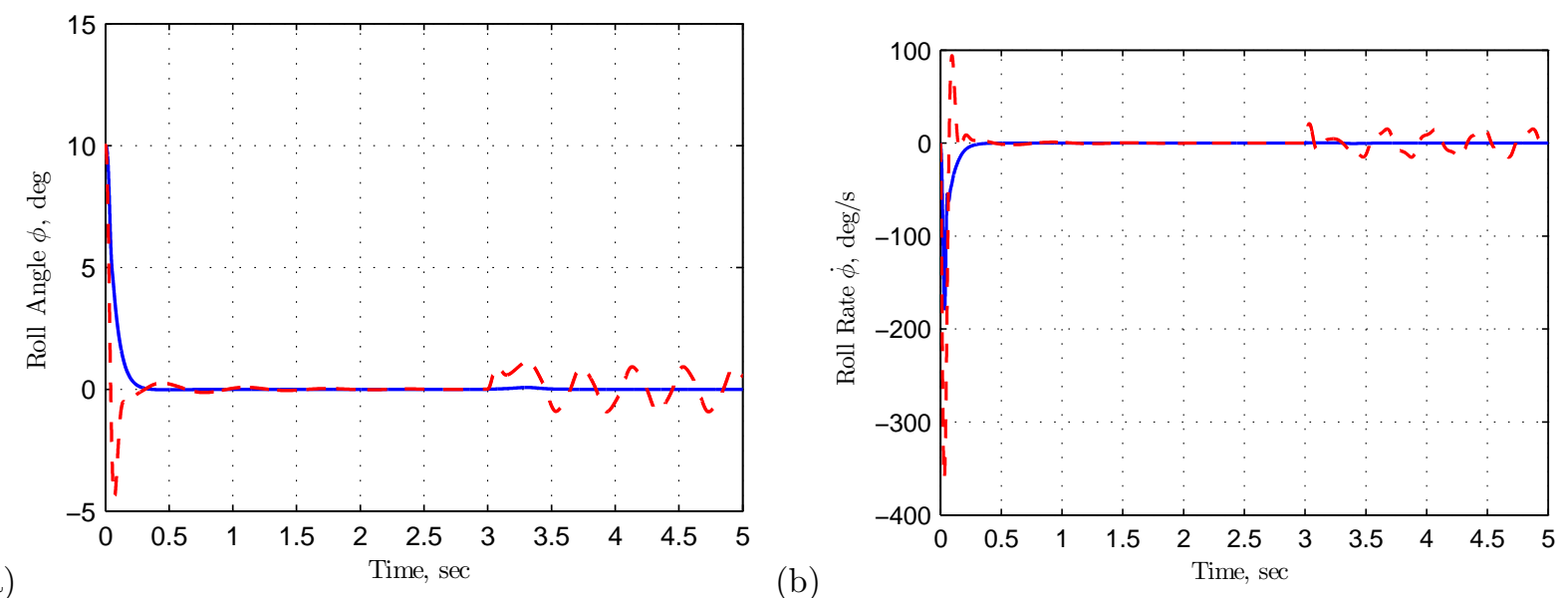

(a)

(b)

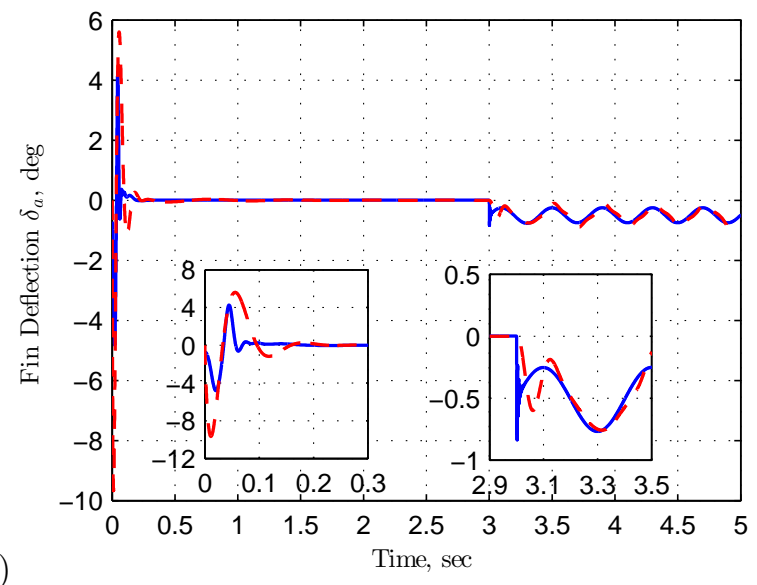

Fig. 3. Robustness performance test results of roll stabilization problem in the presence of disturbances and uncertainties in Case III under the proposed method (solid blue) and the basic ADRC method (dashed red): (a) roll angle; (b) roll rate; (c) fin deflection.

[22] Z. Yang, H. Tsubakihara, S. Kanae, K. Wada, and C. Su. A novel robust nonlinear motion controller with disturbance observer. IEEE Transactions on Control Systems Technology, 16(1):137-147, 2008.

[23] Y. Xia, P. Shi, G.P. Liu, D. Rees, and J. Han. Active disturbance rejection control for uncertain multivariable systems with time-delay. IET Control Theory and Applications, 1(1):75-81, 2007.

[24] J. Ishikawa. A novel add-on compensator for cancelation of pivot nonlinearities in hard disk drives. IEEE Transactions on Magnetics, 34(4):1895-1897, 1998.

[25] S.H. Li and Z.G. Liu. Adaptive speed control for permanent magnet synchronous motor system with variations of load inertia. IEEE Transactions on Industrial Electronics, 56(8):3050-3059, 2009.

[26] Y. Xia, L. Dai, M. Fu, C. Li, and C. Wang. Application of active disturbance rejection control in tank gun control system. Journal of the Franklin Institute, 351(4):2299-2314, 2014.

[27] L. Guo and W.-H. Chen. Disturbance attenuation and rejection for systems with nonlinearity via DOBC approach. International Journal of Robust and Nonlinear Control, 15(3):109-125, 2005.

[28] H. Sun, S. Li, J. Yang, and L. Guo. Non-linear disturbance observer-based back-stepping control for airbreathing hypersonic vehicles with mismatched disturbances. IET Control Theory and Applications, 8(17):1852-1865, 2014.

[29] H. Sun and L. Guo. Composite adaptive disturbance observer based control and back-stepping method for nonlinear system with multiple mismatched disturbances. Journal of the Franklin Institute, 351(2):1027-1041, 2014.

[30] X.J. Wei and L. Guo. Composite disturbance-observer-based control and H-infinity control for complex continuous models. International Journal of Robust and Nonlinear Control, 20(1):106-118, 2010. 
[31] X.J. Wei and L. Guo. Composite disturbance-observer-based control and terminal sliding mode control for non-linear systems with disturbances. International Journal of Control, 92(6):1082-1098, 2009.

[32] Z. Ding. Asymptotic rejection of unknown sinusoidal disturbances in nonlinear systems. Automatica, 1(43):174-177, 2007.

[33] Z. Ding. Asymptotic rejection of unmatched general periodic disturbances with nonlinear Lipschitz internal models. International Journal of Control, 2(86):210-221, 2013.

[34] Z. Ding. Global stabilization and disturbance suppression of a class of nonlinear systems with uncertain internal model. Automatica, 3(39):471-479, 2003.

[35] H.K. Khalil. Nonlinear Systems, 2nd ed. Prentice-Hall, 1996.

[36] Z. Ding. Semi-global stabilisation of a class of non-minimum phase non-linear output-feedback systems. IEE Proceedings-Control Theory and Application, 4(152):460-464, 2005.

[37] C. Qian and W. Lin. Output feedback control of a class of nonlinear systems: a nonseparation principle paradigm. IEEE Transactions on Automatic Control, 10(47):1710-1715, 2002.

[38] S.H. Li, J. Yang, W.-H. Chen, and X.S. Chen. Generalized extended state observer based control for systems with mismatched uncertainties. IEEE Transactions on Industrial Electronics, 59(12):4792-4802, 2012.

[39] F.W. Nesline, H.B. Wells, and Zarchan P. Combined optimal/classical approach to robust missile autopilot design. Journal of Guidance Control and Dynamics, 4(3):316-322, 1981. 


\section{Appendix: Proof of Proposition 1}

The detailed proof of Proposition 1 is presented as follows

$$
\begin{aligned}
& \frac{d}{d t}\left(\frac{1}{2} \zeta_{k+1}^{2}+\frac{1}{2 \gamma_{k+1}} \tilde{d}_{k+1}^{2}\right) \\
& =\zeta_{k+1}\left[\zeta_{k+2}+\alpha_{k+1}+\omega_{o}^{k+1} l_{k+1}\left(y-p_{1}\right)-\dot{\alpha}_{k}\right] \\
& -\gamma_{k+1}^{-1} \tilde{d}_{k+1} \dot{\hat{d}}_{k+1} \\
& =\zeta_{k+1}\left[\zeta_{k+2}-c_{k+1} \zeta_{k+1}-\zeta_{k}-d_{k+1}\left(\frac{\partial \alpha_{k}}{\partial y}\right)^{2} \zeta_{k+1}\right. \\
& -\frac{\partial \alpha_{k}}{\partial y}\left(\psi^{\top} e-\tilde{\psi}^{\top} \xi+\psi^{\top} G \zeta_{1}+\omega_{o} \varepsilon_{2}\right) \\
& \left.-\frac{\partial \alpha_{k}}{\partial \hat{\psi}}\left(\dot{\hat{\psi}}-\tau_{k+1}\right)+\sum_{j=1}^{k} \frac{\partial \alpha_{j-1}}{\partial \hat{\psi}} \Gamma \frac{\partial \alpha_{k}}{\partial y} \xi \zeta_{j}\right] \\
& \leq \zeta_{k+1}\left[\zeta_{k+2}-c_{k+1} \zeta_{k+1}-\zeta_{k}-d_{k+1}\left(\frac{\partial \alpha_{k}}{\partial y}\right)^{2} \zeta_{k+1}\right. \\
& \left.-\frac{\partial \alpha_{k}}{\partial \hat{\psi}}\left(\dot{\hat{\psi}}-\tau_{k+1}\right)+\sum_{j=1}^{k} \frac{\partial \alpha_{j-1}}{\partial \hat{\psi}} \Gamma \frac{\partial \alpha_{k}}{\partial y} \xi \zeta_{j}\right] \\
& -\frac{\partial \alpha_{k}}{\partial y}\left(\psi^{\top} e-\tilde{\psi}^{\top} \xi+\psi^{\top} G \zeta_{1}+\omega_{o} \varepsilon_{2}\right) \zeta_{k+1} \\
& \leq \zeta_{k+1}\left[\zeta_{k+2}-c_{k+1} \zeta_{k+1}-\zeta_{k}-d_{k+1}\left(\frac{\partial \alpha_{k}}{\partial y}\right)^{2} \zeta_{k+1}\right. \\
& \left.-\frac{\partial \alpha_{k}}{\partial \hat{\psi}}\left(\dot{\hat{\psi}}-\tau_{k+1}\right)+\sum_{j=1}^{k} \frac{\partial \alpha_{j-1}}{\partial \hat{\psi}} \Gamma \frac{\partial \alpha_{k}}{\partial y} \xi \zeta_{j}\right] \\
& +\kappa_{2, k+1} e^{\top} e+\frac{\|\psi\|^{2}}{4 \kappa_{2, k+1}}\left(\frac{\partial \alpha_{k}}{\partial y}\right)^{2} \zeta_{k+1}^{2}+\tilde{\psi}^{\top} \frac{\partial \alpha_{k}}{\partial y} \xi \zeta_{k+1} \\
& +\kappa_{3, k+1}\left|\psi^{\top} G\right|^{2} \zeta_{1}^{2}+\frac{1}{4 \kappa_{3, k+1}}\left(\frac{\partial \alpha_{k}}{\partial y}\right)^{2} \zeta_{k+1}^{2} \\
& +\kappa_{1, k+1}\left(\frac{\partial \alpha_{k}}{\partial y}\right)^{2} \zeta_{k+1}^{2}+\frac{\omega_{o}^{2}}{4 \kappa_{1, k+1}} \varepsilon^{\top} \varepsilon \\
& \leq \zeta_{k+1}\left[\zeta_{k+2}-c_{k+1} \zeta_{k+1}-\zeta_{k}-\frac{\partial \alpha_{k}}{\partial \hat{\psi}}\left(\dot{\hat{\psi}}-\tau_{k+1}\right)\right. \\
& \left.+\sum_{j=1}^{k} \frac{\partial \alpha_{j-1}}{\partial \hat{\psi}} \Gamma \frac{\partial \alpha_{k}}{\partial y} \xi \zeta_{j}\right]+\kappa_{2, k+1} e^{\top} e+\tilde{\psi}^{\top} \frac{\partial \alpha_{k}}{\partial y} \xi \zeta_{k+1} \\
& +\kappa_{3, k+1}\left\|\psi^{\top} G\right\|^{2} \zeta_{1}^{2}+\frac{\omega_{o}^{2}}{4 \kappa_{1, k+1}} \varepsilon^{\top} \varepsilon-\delta_{1, k+1}\left(\frac{\partial \alpha_{k}}{\partial y}\right)^{2} \zeta_{k+1}^{2} .
\end{aligned}
$$

